\title{
Erratum to: An adaptive weak continuous Euler-Maruyama method for stochastic delay differential equations
}

\author{
B. Akhtari • E. Babolian • A. Foroush Bastani
}

Published online: 18 February 2015

(C) Springer Science+Business Media New York 2015

\section{Erratum to: Numer Algor (2014) \\ DOI 10.1007/s11075-014-9880-6}

- In Section 2, the sentence "We notice that the choice of $\theta=1$ gives us the discrete Euler-Maruyama scheme." has to be removed.

- In the proof of Theorem 3.2, the interval $[0, T]$ has to be replaced by $\left[t_{0}, T\right]$.

- At the end of the proof of Theorem 3.2, the notations $\psi_{N_{0}-1}$ and $h$ have to be replaced by $\widetilde{\psi}_{N_{0}-1}$ and $|\Lambda|$.

- In Section 3, after Remark 3.4, "E্E( $\left(f\left(X\left(t_{0} ; \psi\right)\right)\right)$ " has to be replaced by " $\mathbb{E}\left(f\left(\widetilde{X}^{t_{0}, X_{0}}(T ; \widetilde{\psi})\right)\right)$ ".

- At the beginning of Section 4, before Lemma 4.1, we should have "We use the notation $\partial_{\alpha}$ for denoting $\partial_{i_{1} i_{2} \ldots i_{n}}$ with $i_{j} \in\{1,2,3, \ldots, d\}$ and $|\alpha|=n$ for the number of differentiations.", instead of what is written.

- In Section 4, after the proof of Lemma 4.1, we should have "Considering (4.1) for $f \in C_{P}^{m_{0}}\left(\mathbb{R}^{d}, \mathbb{R}\right)$ with $m_{0} \geq 8$, if we replace $\partial_{\alpha} u$ by $\partial_{\alpha} \bar{u}$ in (3.14), the order of convergence does not change and a new expansion is obtained as..." instead of what is written in the paper.

The online version of the original article can be found at http://dx.doi.org/10.1007/s11075-014-9880-6.

\footnotetext{
B. Akhtari $(\bowtie) \cdot$ E. Babolian

Department of Mathematical Sciences and Computer, Kharazmi University,

50 Taleghani Avenue, Tehran 1561836314, Iran

e-mail: Akhtari@tmu.ac.ir; Akhtaribahareh44@gmail.com

E. Babolian

e-mail: babolian@saba.tmu.ac.ir
}

A. Foroush Bastani

Department of Mathematics, Institute for Advanced Studies in Basic Sciences,

P. O. Box 45195-1159, Zanjan, Iran

e-mail: bastani@iasbs.ac.ir 
- In Section 5, at the first step, replace "TOLS $=\frac{1}{9}$ TOL" by "TOLTS $=\frac{1}{9}$ TOL".

- At the end of Section 5, the command "E $\left(f\left(\tilde{X}(T ; \widetilde{\psi}), M_{c}[0]\right)\right)=2$ TOL" has not been used in our program. Moreover, replace twice $\mathbf{E}\left(f\left(\widetilde{X}(T ; \widetilde{\psi}), M_{c}[0]\right)\right)$ by $\mathbf{E}\left(f\left(\widetilde{X}(T ; \widetilde{\psi}), M_{c}[k]\right)\right)$. 